\title{
Implementation of a Medical Database System for a Radiosurgery Center
}

\author{
Markus Kufeld ${ }^{1}$, Christoph Fürweger ${ }^{2}$, Christian G. Drexler ${ }^{3}$, B. Wowra ${ }^{4}$, Alexander \\ Muacevic ${ }^{5}$ \\ 1. Charité Cyberknife Center Berlin 2. Chief Medical Physicist, European CyberKnife Center Munich 3. \\ Varian Medical Systems International 4. European Cyberknife Center Munich-Grosshadern 5. European \\ CyberKnife Center Munich
}

$\square$ Corresponding author: Markus Kufeld, mqfeld@mac.com

Disclosures can be found in Additional Information at the end of the article

\section{Abstract}

Objective: To develop and integrate a database system into the heterogeneous network environment of a dedicated radiosurgery treatment center with the goal to facilitate documentation, quality assurance, patient care, and scientific research.

Methods: We customized a database on the basis of the commercial software FileMaker Pro ${ }^{\circledR}$ (FileMaker Inc., Santa Clara, CA). The multi-dimensional database system was fully integrated into the network infrastructure of the center. Automated data import and export was established utilizing programmed scripts. Specially trained medical assistants are in charge of the data management.

Results: The database stores medical information prospectively and integrates all data of the radiosurgical process, from basic patient data to treatment parameters and follow-up information. All information is quickly available at any workplace in the center. Continuous adaptation of the data management to daily workflows ensures the accuracy and validity of the stored information. Automated scripts generate up-to-date reports and statistical analyses.

Conclusions: The database enhances the quality of documentation, treatment, and patient care as well as comfort and efficiency in daily information workflows. To achieve this, the allocation of personnel with responsibility for the database system as well as the data management is crucial.

Categories: Medical Education, Radiation Oncology, Neurosurgery

Keywords: medical database, radiosurgery

\section{Introduction And Background}

\section{Published 12/09/2009}

\section{(c) Copyright 2009}

Kufeld et al. This is an open access article distributed under the terms of the Creative Commons Attribution License CC-BY 3.0., which permits unrestricted use, distribution, and reproduction in any medium, provided the original author and source are credited.
The management of medical patient information in an electronic database system should offer an efficient data storage and retrieval. Pre-designed electronic health record solutions do not meet the needs of specialized institutions, cannot be customized and may induce high costs.

These systems set priorities to practice organization and reimbursement, but cannot handle clinical information sufficiently.

Therefore, we created computerized information system based on a commercially available DBMS. The implementation of this medical database system contained three steps: the 
planning and development of the database, the integration into existing network structures, and the continuous adaptation of the data management to daily workflows.

Our database had to fulfill several conditions: it was planned to store medical patient data prospectively and integrate all information of the radiosurgical process, from basic patient data to treatment parameters and follow-up information.

The system had to be user-friendly, scalable and compatible with the heterogeneous practice network infrastructure. Additionally, it should provide standardized reports and export datasets for statistical analyses.

We here describe the customized database system and its integration into the network infrastructure. We analyze the data management and workflows emphasizing their importance for the validity and quality of the database. Problems that occurred during the process of implementation and possible solutions are discussed.

\section{Abbreviations}

XML eXtensible Markup Language

XSLT eXtensible Stylesheet Language Transformation

DBMS Database Management System

CT Computer Tomography

PACS Picture Archiving and Communication System

LAN Local Area Network

RTOG Radiation Therapie Oncology Group

AVM Arterio-Venous Malformation

\section{Review}

\section{Methods}

The database system FileMaker Pro $8.5{ }^{\circledR}$ (FileMaker, Inc., Santa Clara, CA) serves as a basic platform. The commercial software package combines a flexible and scalable database with a convenient user interface. A simple script language enables the creation scripts for queries, work routines or data export. The system is compatible with the Mac and Windows operating systems.

The DBMS allows a server/client architecture, which had to be integrated into the network environment. This network includes the patient management system, containing basic patient information, a CT scanner connected to a PACS, and the radiosurgery system itself. The radiosurgical hardware comprises the treatment planning workstations (Multiplan ${ }^{\circledR}$ ) as well as the treatment system itself. For security reasons, all components connected with the treatment process are established in an independent parallel local area network.

Interfaces had to be adjusted to support data import from the preexisting networks and export for statistical analyses. 


\section{Cureus}

Multi-dimensional database system

As a relational database, FileMaker Pro ${ }^{\circledR}$ interconnects two-dimensional tables to a multidimensional system (Figure 1). Corresponding tables are interconnected using primary key fields. The database establishes five major tables with a varying number of attributes:

\section{Basic patient information}

The basic patient data is imported from the practice management system and updated on a daily basis. The patient table contains data that occurs only once per patient (e.g. insurance, date of birth, date of death). This table is linked by a "one-to-many" connection with the medical information table. Each patient can have none, one or multiple datasets representing a treatment session.

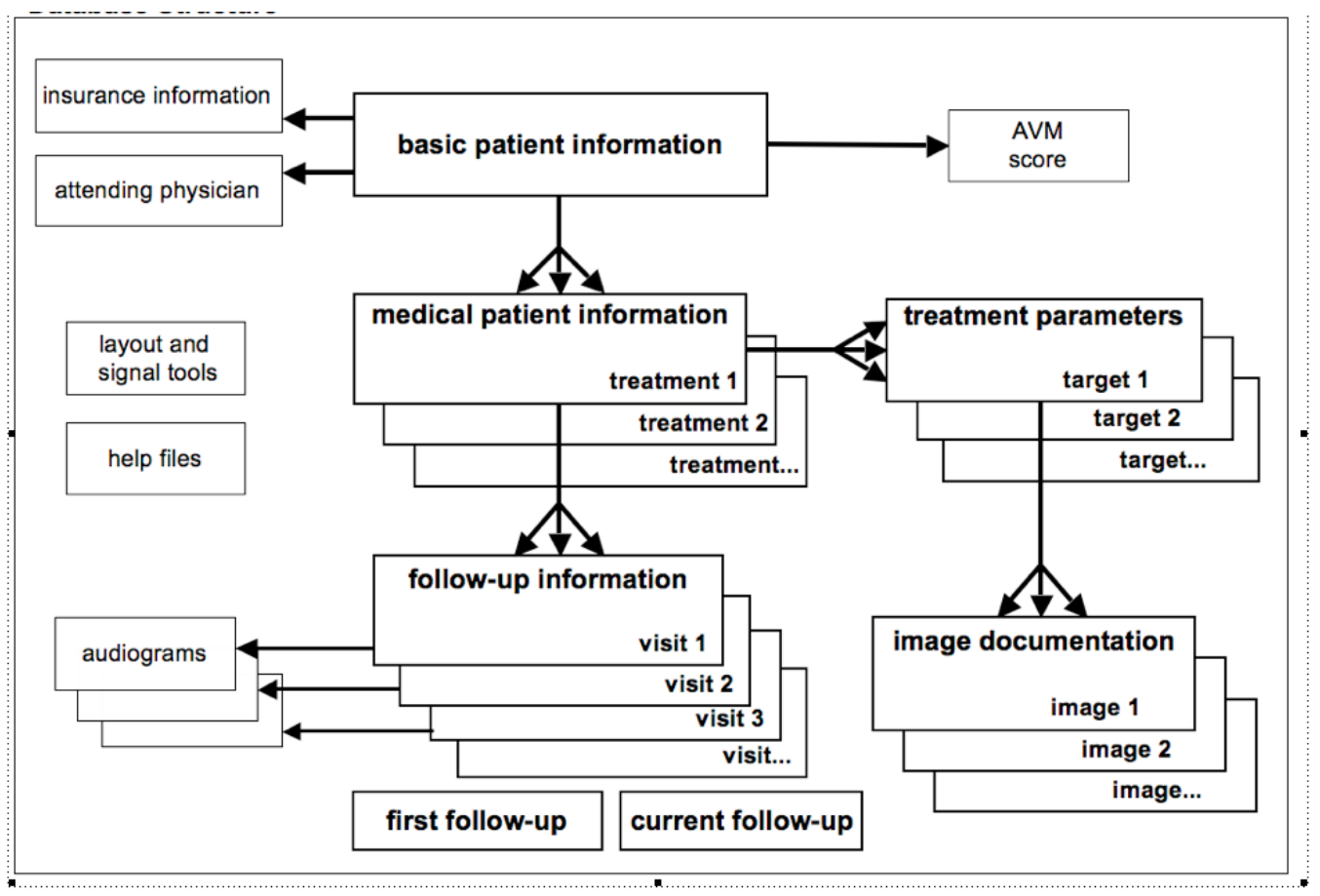

FIGURE 1: Database Structure

\section{Medical patient information}

The medical and clinical information of a patient corresponds to his current treatment. One patient may have multiple treatments. Each treatment has its own series of follow-up, which is stored in the follow-up table.

\section{Follow-up information}

Each treatment can be connected to multiple follow-up datasets in a timely sequence. The medical status at follow-up visit, evaluation of radiological imaging, RTOG, and quality of life scoring are stored in the follow-up table. 


\section{Cureus}

\section{Treatment parameters}

The technical parameters of the treatment are linked to the medical information table. Each treatment can contain multiple datasets, for example if multiple targets are treated in one session. One patient can have multiple treatment parameter sets. The data is extracted via XSLT transformation from the treatment plan XML file.

Image documentation

The image table is also linked to the medical information table. Representative plan screenshots are imported from the planning workstations and conjoined with the treatment. The image table can also store media data like pictures, scans, audio files, or movie clips.

\section{Minor relations}

Besides the five major relations, there are additional tables: insurance information, attending physician, audiogram, AVM score, patient satisfaction questionnaire, and explaining help texts.

User-defined layouts

The layouts (views) serve as the user interface to the database. A view defines the display of information to the user. With views, the data can be filtered and values from different tables are summarized. The main layouts are arranged as clickable tabs to facilitate access to different layouts.

\section{Main Layouts}

\section{Patient information overview}

Clinical information

Treatment information

Follow up information

Plan screenshots

\section{Additional Layouts}

Target information overview

Audiograms

Vestibular schwannoma classification

AVM classification

Image overview

Anatomical regions

Health insurance

Patient satisfaction questionnaire

Help window

Reports

TABLE 1: Layouts 
The DBMS offers a user-friendly scripting tool. Basic functions can be put together with a point-and-click approach. Complex scripts make use of variables, loops, or mathematical functions. Scripts functions are connected to buttons in the layouts to open, close, or change between layouts or tabs. The script jobs are triggered by these buttons or run automatically in the background. The data import and export is accomplished with scripts as well. Automated scripts create data overviews and summaries. For key indications, scripts are predefined to conduct complex queries and to transfer the data directly to the statistical analysis software.

\section{Results}

Database implementation

A software company developed the initial database system. Because of the lack of experience in the medical field, the provided draft of a database did not meet the expectations and needs of the users. Subsequently, a physician with knowledge in medical informatics was consigned to reconstruct the database and to optimize it according to values, parameters, granularity, and usability.

A detailed documentation of the database structure, implementation, data workflow, and functions was compiled. All programmed scripts are listed and commented. Constant advancements took place under close consultation with the users.

\section{Data management}

Since implementation in April 2006, the database integrates more than 25,000 datasets to date, containing all clinical and treatment information of all treated patients as well as their current follow-up information.

The starting point of data entry is the import of basic patient information from the patient management system. This import via one-click is conducted by a script that updates the patient information and enters new patients to the patient data table. The basic patient data is registered initially at the counter and entered into the practice information system. This is done by reading-out the patient's health insurance card or manually by the employee.

The medical data concerning the current treatment and medical history encompass the important clinical information. The values are extracted from the patient health record and entered manually into the database system. Medically trained personnel are in charge of the data entry, which is carried out during the patient treatment. Free text entry is limited. Repeated values are stored in value lists and are accessible by drop down menus.

The technical treatment parameters are drawn from the operation system after the treatment and imported into the database as XML file using a XSLT transformation script.

The follow-up assessment is registered in the patient chart by the consulting doctor and transferred to an employee who is in charge of collecting and entering all follow-up information (Figure 2). 


\section{Cureus}

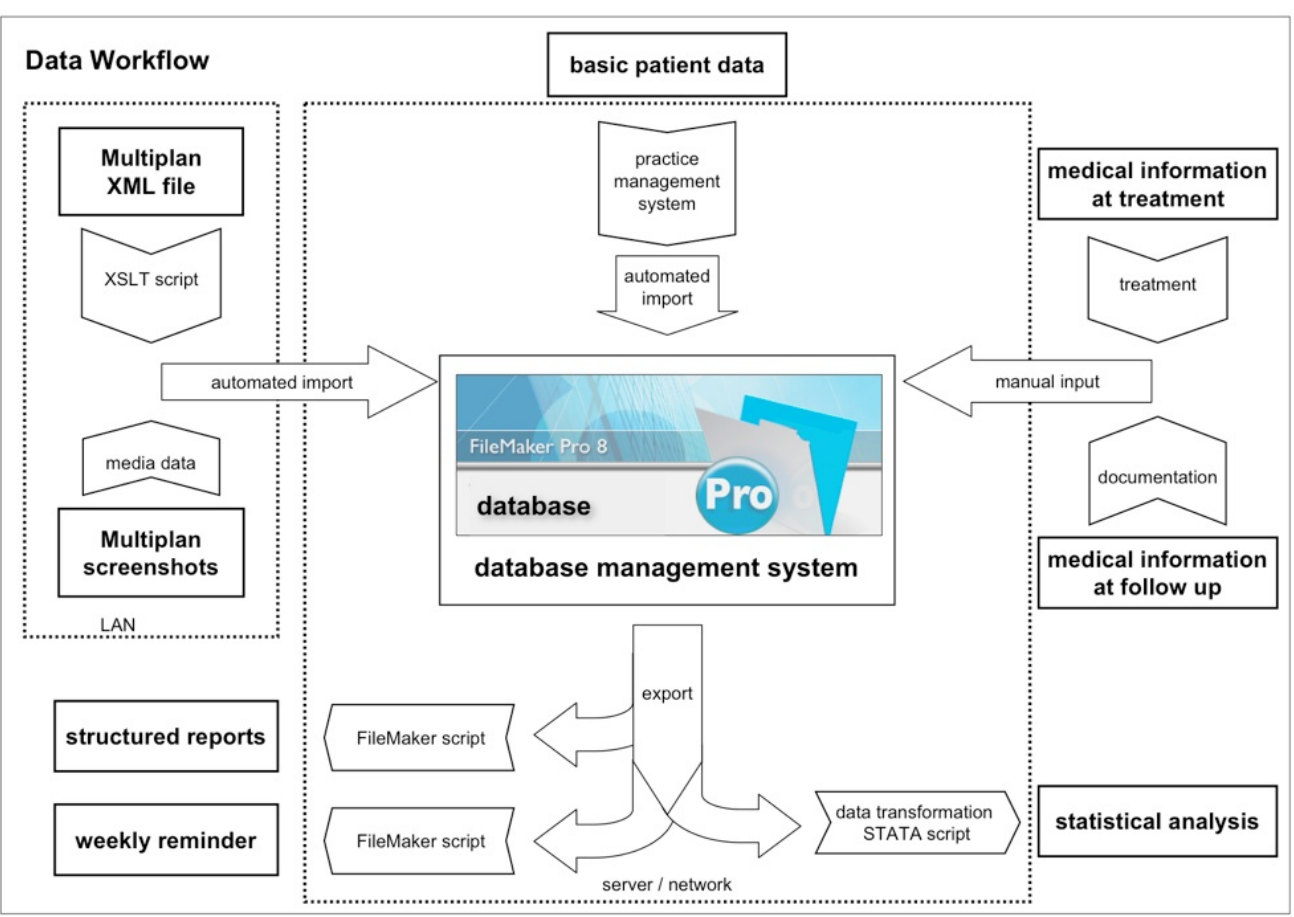

FIGURE 2: Data Workflow Scheme

\section{Data workflow}

An assistant medical technician enters the patient's medical information during the treatment, so the database contains all baseline information at the end of the treatment without delay. The accumulation of patient charts that have to be processed is avoided.

The prompt entry of the follow-up information is integrated into the reporting workflow: after the follow-up examination a report is created for the patient as well as the attending family doctor. A trained medical transcriptionist coordinates the report writing and enters all relevant follow-up information into the database system. A flag is set in the database system to schedule the next follow-up visit. If the patient should exceed the next follow-up date by two months, the weekly reminder list brings up all overdue follow-up visits.

Finally, the treatment parameters, physical and technical values are extracted from the treatment plan files and added to the patient dataset.

\section{Performance of the database system}

1. The installation with multiple clients and one server offers a high availability of the database system at different workstations and workplaces. The efficiency of the data management is enhanced because the data input can be conducted independently from every workplace in the center.

To ensure data safety, a sequence of back-ups is generated hourly, daily and monthly and stored on independent hard drives.

2. The data management workflow provides a high grade of completeness and information 
integrity. Restrictions during the data entry minimize the input of false values.

The prompt data entry ensures the actuality of the stored information.

3. The quality and reliability of captured information is enhanced, because the information is handled with knowledge of anatomical and medical contexts by assistant medical technicians with a clinical background.

4. The DBMS allows the adaptation to changing structures and tasks. Newly requested storage and analysis options can be established on the go. The continuous optimization adjusts the system closely to the users' needs.

\section{Discussion}

Scientific papers concerning medical database systems mainly analyze health information technology in general or describe specific, individually developed database systems [1-10].

The impact of health IT in general on quality, efficiency, and costs was analyzed by Chaudhry, et al. Their systematic review revealed the efficacy of health IT in improving quality and efficiency. However, this was found to be true only for a few institutions, because the analyzed systems were heterogeneous and hardly comparable [11].

Johnson and Ventura confirmed the general benefits of health IT solutions, improving patient safety and quality care for their large institution [12].

A description of a specific database system for surgical outcomes was published by Newcomb, et al. They demand a combination of clinical informatics and research expertise to develop a robust surgical outcomes database [13].

A similar database to our system is called AdOnco, which is presented by Hambek, et al. The authors developed a head and neck oncology database for clinical and scientific purposes, which was integrated into a patient documentation system. The combination of clinical and scientific features proved to be very effective in daily routine and research [14].

In comparison to the described database systems, we analyzed the specific benefits and bottlenecks of our system.

\section{Benefits}

The database system provides benefits for the data workflows, for the quality of patient care and for scientific research:

\section{Benefits for data workflows and documentation}

The network structure allows access to the database system from every workplace in the treatment center. The database system itself offers quick access to all patient and treatment information, plan screenshots, and medical documentation. The clearly defined data workflows keep the information up to date. Queries and data retrieval for quality assurance, reimbursement issues or health insurances are facilitated.

Benefits for the quality of patient care 
The clearly structured data collection allows a quick survey over all medical information of the patient. All data is right at hand during the follow-up examination and is accessible at any time from every workplace.

The attending physician has more information right at hand and can concentrate on the patient to deliver a service with higher quality.

\section{Benefits for scientific research}

The prospective data collection delivers complete, accurate and valid data. The clearly organized and structured data input, the follow-up reminders, and the continuous data control warrants the quality of the data collection.

The compilation of script routines for an automated export of complex queries facilitates the extraction of large datasets. These datasets are processed for predefined statistical analyses. All statistical analyses are standardized.

The database serves as a broad informational basis for scientific questions, integrating all relevant medical and technical information in one system.

\section{Data management}

Besides the development and implementation of the software, the adoption of the data management (data entry) into the daily workflows was crucial.

Depending on the amount of data as well as the complexity of the database system, a dedicated data manager or administrator is mandatory. If no extra personnel can be allocated for this, at least a coordinator is needed to organize the data input and fit the workflow to the existing workforce. A delay in data input results in a demanding accumulation of data that has to be processed.

In 1988, Dambro, et al. published their unsuccessful trial to implement a computerized medical record system. The personnel expenses to assure the data entry overdrew the budget only four months after implementation [15].

In our center, a high value was set on the database project and sufficient personnel was allocated for the data management.

However, three main problem areas remained to be solved:

1. Assurance of complete and correct data entry.

2. Complex data export from multi-dimensional database.

3. Limited in-built statistical capacities of the DBMS.

\section{Automated data import}

The information retrieval takes place at several workplaces and procedures of the treatment center. Initially, the basic patient data is registered at the front desk and entered into the practice information system. The patient's health insurance card is read out or data has to be entered manually by the front desk manager. To avoid a redundant data entry, the patient's 
basic data is imported automatically off from the practice information system. An interface accesses the database of the practice management system (dBase format) and imports the matching field values into the FileMaker patient information table. New patients are added as new datasets, existing dataset are updated.

The treatment information is as well automatically imported from the CyberKnife operation system. The treatment system generates an XML plan file containing all treatment parameters. The size of this file can extend to 20,000 pages. To import the XML file into the FileMaker system, the plan XML file is transformed into a FileMaker-readable XML file by an XSLT script. The relevant treatment parameters can be extracted from the XML file and are assigned to the values of the database treatment parameters table. The XSLT script is easily adjustable to any changes in the treatment XML file.

\section{Data accuracy}

The correct data entry depends on the proper extraction of information from the patient charts, as already stated by Hogan, et al. in 1997 [16]. To facilitate the collection of information, our attending physicians use a standardized protocol sheet on which the examination and followup results are compiled.

A continuous training of the employees who are in charge of the data input warrants the data integrity. To assure correct data, entry field type definitions restrict the data type to numbers, text, dates, or media. The entered values are checked automatically for their data type, and the input is blocked if not matching the field definition. Additionally, the administrator checks the tables for missing or outstanding values on a weekly basis.

A prompt data input enhances the quality of information and the validity of queries that are conducted. Kush, et al. confirmed the impact of direct point-of-care data capture on the quality of data [17].

A delay in the data workflow leads to an accumulation of charts to be processed and can cause errors during the tedious data input.

\section{Complex data queries}

The usability of the database is facilitated by the multi-dimensional design with linked tables. A connection of one dataset in a table to many datasets of another table, e.g. one patient with multiple treatments and each treatment with multiple follow-up datasets, is called a "one to many" (1:n) connection. This multi-dimensional structure of the database eases the survey and understanding of the database. On the other hand, statistical software for complex statistical analysis usually works with two-dimensional tables.

When conducting a database query, the multidimensional structure has to be transformed into a two-dimensional table. The transformation requires some understanding of the principal database structure. The common user will not be able to generate a complex query from a relational database structure. At this point, the administrator has to create the accurate query construction.

The multi-dimensional structure guarantees the comfortable data input and convenient daily use of the database, but causes difficulties in complex or customized queries that can only be handled by users who are familiar with the underlying database structure.

\section{Limited statistical capabilities}


The reason for creating customized queries is the limited capability of the DBMS to solve elaborated statistical tasks. The scientific use of the medical database requires reiterated extractions of complex datasets. The datasets have to be prepared for statistical analyses. This can be awkward, especially with large datasets containing many variables.

We established an automated transcription algorithm: with the scripting language of the statistics software (STATA Inc., Texas) specific scripts (do-files) were created to accomplish the transformation of the exported FileMaker data to a STATA compatible dataset. The STATA scripts are allocated for different queries. They do not only prepare the data for import but also provoke pre-defined statistical operations to generate a standardized set of statistical results for similar or repeated queries.

Although the basic statistical analysis is automated by scripting, any changes or special requests will call for the database administrator or programmer to customize the query structure, script structure or statistical evaluation according to the user's requests.

\section{Conclusions}

The predefined aims of the database could be achieved after a period of continuous optimization of the system.

The database system supports the prospective storage of medical patient information and integrates all relevant information of the radiosurgical process.

The heterogeneous network infrastructure could be integrated. The medical practice management system as well as the radiosurgery system is connected to the database system. This is achieved by customized interfaces for data import and export.

Standardized surveys and statistical reports are generated using FileMaker scripts as well as customized scripts for statistical analyses.

The database emerged as a valuable tool to integrate the information management into the daily workflows. The DBMS enhances the quality of documentation, treatment, and patient care as well as comfort and efficiency in daily information workflows. To achieve this, dedicated personnel have to be responsible for the database system as well as the data management.

\section{Additional Information}

\section{Disclosures}

Conflicts of interest: In compliance with the ICMJE uniform disclosure form, all authors declare the following: Payment/services info: All authors have declared that no financial support was received from any organization for the submitted work. Financial relationships: All authors have declared that they have no financial relationships at present or within the previous three years with any organizations that might have an interest in the submitted work. Other relationships: All authors have declared that there are no other relationships or activities that could appear to have influenced the submitted work.

\section{References}

1. Birch DW, Park A, Bailey M, Witzke W, Witzke D, Hoskins J: The development and implementation of a computerized database for clinical research in minimal access surgery. An international pilot study. Surg Endosc. 2001, 15:1008-1010.

2. Brandt CA, Deshpande AM, Lu C, Ananth G, Sun K, Gadagkar R, Morse R, Rodriguez C, Miller 
PL, Nadkarni PM: TrialDB: A web-based Clinical Study Data Management System. AMIA Annu Symp Proc. 2003. 794.

3. Brown PJ, Sonksen P: Evaluation of the quality of information retrieval of clinical findings from a computerized patient database using a semantic terminological model. J Am Med Inform Assoc. 2000, 7:392-403.

4. Fisk JM, Mutalik P, Levin FW, Erdos J, Taylor C, Nadkarni P: Integrating query of relational and textual data in clinical databases: a case study. J Am Med Inform Assoc. 2003, 10:21-38.

5. Kahn MG: Three perspectives on integrated clinical databases . Acad Med . 1997, 72:281-286.

6. Meystre S: Electronic patient records: some answers to the data representation and reuse challenges. Findings from the section on Patient Records. Yearb Med Inform. 2007, 47-49.

7. Mosis G, Vlug AE, Mosseveld M, Dieleman JP, Stricker BC, van der Lei J, Sturkenboom MC: A technical infrastructure to conduct randomized database studies facilitated by a general practice research database. J Am Med Inform Assoc. 2005, 12:602-607.

8. Palta JR, Frouhar VA, Dempsey JF: Web-based submission, archive, and review of radiotherapy data for clinical quality assurance: a new paradigm. Int J Radiat Oncol Biol Phys . 2003, 57:1427-1436.

9. Sailer SL, Tepper JE, Margolese-Malin L, Rosenman JG, Chaney EL: RAPID: An Electronic Medical Records System for Radiation Oncology. Semin Radiat Oncol . 1997, 7:4-10.

10. Schell SR: Creation of clinical research databases in the 21 st century: a practical algorithm for HIPAA Compliance. Surg Infect (Larchmt) . 2006, 7:37-44.

11. Chaudhry B, Wang J, Wu S, Maglione M, Mojica W, Roth E, Morton SC, Shekelle PG: : Systematic review: impact of health information technology on quality, efficiency, and costs of medical care. Ann Intern Med . 2006, 144:742-752.

12. Johnson T, Ventura R: Applied informatics for quality assessment and improvement. J Nurs Care Qual. 2004, 19:100-104.

13. Newcomb WL, Lincourt AE, Gersin K, Kercher K, Iannitti D, Kuwada T, Lyons C, Sing RF, Hadzikadic M, Heniford BT, Rucho S: Development of a functional, internet-accessible department of surgery outcomes database. Am Surg . 2008, 74:548-54.

14. Hambek M, Adunka O, Baghi M, Gstoettner W, Knecht R: AdOnco: a database for clinical and scientific documentation of head and neck oncology. Ann Otol Rhinol Laryngol . 2006, 115:144-149.

15. Dambro MR, Weiss BD, McClure CL, Vuturo AF: An unsuccessful experience with computerized medical records in an academic medical center. J Med Educ . 1988, 63:617-623.

16. Hogan WR, Wagner MM: Accuracy of data in computer-based patient records . J Am Med Inform Assoc . 1997, 4:342-355.

17. Kush R, Alschuler L, Ruggeri R, Cassells S, Gupta N, Bain L, Claise K, Shah M, Nahm M: Implementing Single Source: the STARBRITE proof-of-concept study . J Am Med Inform Assoc. 2007, 14:662-673. 